\title{
Factors predicting the risk of loss of decisional capacity for medical assistance in dying: a retrospective database review
}

\author{
Debbie Selby MD, Christopher Meaney MSc, Sally Bean JD MA, Elie Isenberg-Grzeda MD, \\ Amy Nolen MBBS MSc
}

\section{Abstract}

Background: Bill C-14, the legislation that legalized medical assistance in dying (MAiD) in Canada in 2016, outlines eligibility criteria and includes both a mandated 10-day reflection period and a requirement that the patient have capacity to consent at the time MAiD is provided. We examined clinical factors associated with shortened reflection periods or loss of capacity before provision of MAiD.

Methods: This retrospective database review involved patients who requested MAiD at a tertiary care hospital in Toronto, Canada, between June 2016 and April 2019. We used logistic regression analyses to examine the association between the combined outcome of unanticipated loss of decisional capacity, shortening of the reflection period or death and the clinical risk factors of interest (age, sex, location of MAiD request [inpatient v. outpatient], score on palliative performance scale [PPS] and diagnosis [cancer v. noncancer]). We generated receiver operating characteristic curves to identify the PPS score (encompassing 5 functional domains: ambulation, activity level, self-care, intake and level of consciousness) that best predicted loss of capacity, shortening of the reflection period or death.

Results: In total, 155 patients requested assessment for MAiD, and 136 of these were included in the statistical analyses. For 68 patients, the reflection period was not shortened; the other 68 patients lost capacity, died or required shortening of the reflection period. In contrast to the results for age, sex, location of request and diagnosis, the PPS score was associated with loss of capacity or shortening of the reflection period (odds ratio 4.63, 95\% confidence interval 2.87-8.23, per 10-point decrease in PPS score). PPS scores less than or equal to $40 \%$ balanced sensitivity, specificity and negative predictive value while emphasizing sensitivity to prevent false negative errors.

Interpretation: The PPS score at the time of MAiD request was strongly associated with loss of capacity or shortening of the reflection period, with lower scores incrementally increasing the risk of these outcomes. For patients with a PPS score of $40 \%$ or below, close monitoring is warranted, potentially with plans made to allow rapid provision of MAiD should their clinical condition deteriorate.

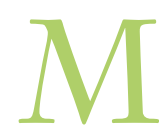
edical assistance in dying (MAiD) was decriminalized by the Supreme Court of Canada in February 2015, ${ }^{1}$ and Canada's federal legislation on MAiD (Bill C-14) was passed on June 17, 2016.' Since the legislation was passed, through to the end of 2019, a total of 13946 Canadians have accessed MAiD. ${ }^{3}$ Canada joined a growing number of international jurisdictions affording individuals the right to an assisted death, including regions in Europe, the United States, Latin America and Australia. ${ }^{4-9}$

In Canada, the eligibility requirements for MAiD stipulate that the patient be at least 18 years of age, make a voluntary request, possess capacity to provide consent for MAiD and have a grievous and irremediable medical condition. The latter is defined as an illness, disease or disability resulting in an advanced state of irreversible decline wherein the patient is experiencing intolerable physical or psychological suffering and natural death has become reasonably foreseeable. Patients deemed eligible for MAiD by 2 independent physicians or nurse practitioners must fulfill a 10 -day reflection period

Competing interests: For activities outside the work reported here, Elie Isenberg-Grzeda has received consultancy fees from Celgene USA. No other competing interests were declared.

This article has been peer reviewed.

Correspondence to: Debbie Selby, debbie.selby@sunnybrook.ca CMAJ Open 2020. DOI:10.9778/cmajo.20200052 
before the procedure is provided and must retain capacity to provide final consent immediately before provision. ${ }^{2}$ Following provision of MAiD, there is mandatory reporting by practitioners at the federal level for monitoring ${ }^{10}$ and at the provincial or territorial level for oversight, with this process varying across the provinces and territories. ${ }^{11}$

Prior work has highlighted the importance that patients place on receiving MAiD once they have made the decision to proceed. ${ }^{12-14}$ For those who are seriously ill or felt to be at risk of losing capacity to provide final consent, the 10-day reflection period can be a source of marked anxiety for the patient, with providers needing to balance patient-centred care with adherence to the legislation. The reflection period can be waived if both assessors feel the patient is at imminent risk of death or loss of capacity, but to date there are no evidence-based data to help assessors determine who might be at such risk. Across Canada, as of Dec. 31, 2019, about one-third of patients $(34.3 \%)$ had a shortened wait time, ${ }^{3}$ but nevertheless there are patients who die or lose capacity before provision of MAiD. ${ }^{12}$ The goal of this study was to identify clinical factors associated with shortening of the reflection period or loss of capacity to consent before provision of MAiD.

\section{Methods}

\section{Study setting and participants}

We reviewed our institutional MAiD database, which includes all patients referred for a MAiD assessment at Sunnybrook Health Sciences Centre, a 638-bed tertiary care hospital in Toronto, Canada, with an affiliated inpatient palliative care unit. The hospital's MAiD service includes a central coordinator and multiple MAiD assessors across various medical specialties. The service conducts MAiD assessments for patients in ambulatory and inpatient settings and provides MAiD to eligible patients. Study participants included any patient who underwent a MAiD assessment between June 2016 and April 2019.

\section{Data sources}

The hospital's MAiD database was created after the enactment of MAiD legislation and is maintained by the hospital's MAiD coordinator. The database captures all patients who request an eligibility assessment at this hospital. Such requests include informal verbal or written requests and formal completion of a signed and witnessed document. Demographic data are recorded at the time of the assessment request.

For this study, we retrospectively expanded the database to include further clinical data using information from the eligibility assessments and the associated electronic patient records. Three of the authors (D.S., E.I.-G., A.N.) extracted the data; a fourth author (S.B.) performed an independent cross-check of a random subset of the data for accuracy.

We collected the following data: date of MAiD request, dates of eligibility assessments, date when the witnessed request was signed, patient demographic characteristics, primary diagnosis relevant to the MAiD request, location where the MAiD request occurred (outpatient, acute care inpatient or palliative care unit inpatient), palliative performance scale (PPS) score at time of first assessment and outcome of the MAiD request. The PPS (Appendix 1, available at www.cmajopen.ca/content/8/4/E825/suppl/DC1) is a validated tool for measurement of a patient's physical status according to 5 functional domains (ambulation, activity level, self-care, intake and level of consciousness); it ranges from $0 \%$ (dead) to $100 \%$ (normal) in increments of 10 percentage points. ${ }^{15,16} \mathrm{We}$ classified the primary diagnosis as either cancer or noncancer. Noncancer diagnoses included cardiovascular or respiratory disease, neurodegenerative disease and other nonmalignant illnesses.

\section{Outcome measures}

Each participant placing a MAiD request underwent 2 independent eligibility assessments and signed a formal, witnessed written request. Signing of the witnessed written request triggered initiation of the 10 -day reflection period. During this period, if both assessors agreed that death or loss of capacity to consent was imminent, the reflection period was shortened (identified as a binary value: shortened v. not shortened). The outcome of the MAiD request was classified as follows: patient received MAiD after completion of the reflection period (group 1); patient received MAiD but with shortening of the reflection period (group 2a); or patient did not receive MAiD, even though a date had been established, because of loss of capacity to consent (occurring within the reflection period) or unanticipated death within the reflection period (group 2b).

\section{Statistical analysis}

We analyzed the demographic and clinical variables using descriptive statistics.

To determine potential predictors of a shortened reflection period, we performed bivariable and multivariable logistic regression analyses (using SAS software, version 9.4 for Windows, SAS Institute Inc.) to test for association of the following clinical risk factors with the outcomes of shortened reflection time or loss of decisional capacity or death during the reflection period: age, sex, location of the MAiD request (inpatient $\mathrm{v}$. outpatient), PPS score and diagnosis (cancer v. noncancer).

Given the goal of identifying risk factors for patients at risk of losing capacity in the 10-day reflection period, the study groups for regression analysis were defined as follows (Figure 1): group 1, patients who received MAiD after completing the 10-day reflection period; and group 2, patients who had their reflection period shortened by the primary or secondary assessors (i.e., because they were felt to be at imminent risk of losing capacity) and patients who actually did lose capacity or died unexpectedly within the 10-day reflection period.

The rationale for group 2 was to capture all patients who actually lost capacity or died, as well as those thought to be at imminent risk of losing capacity or dying, to build a suitable 


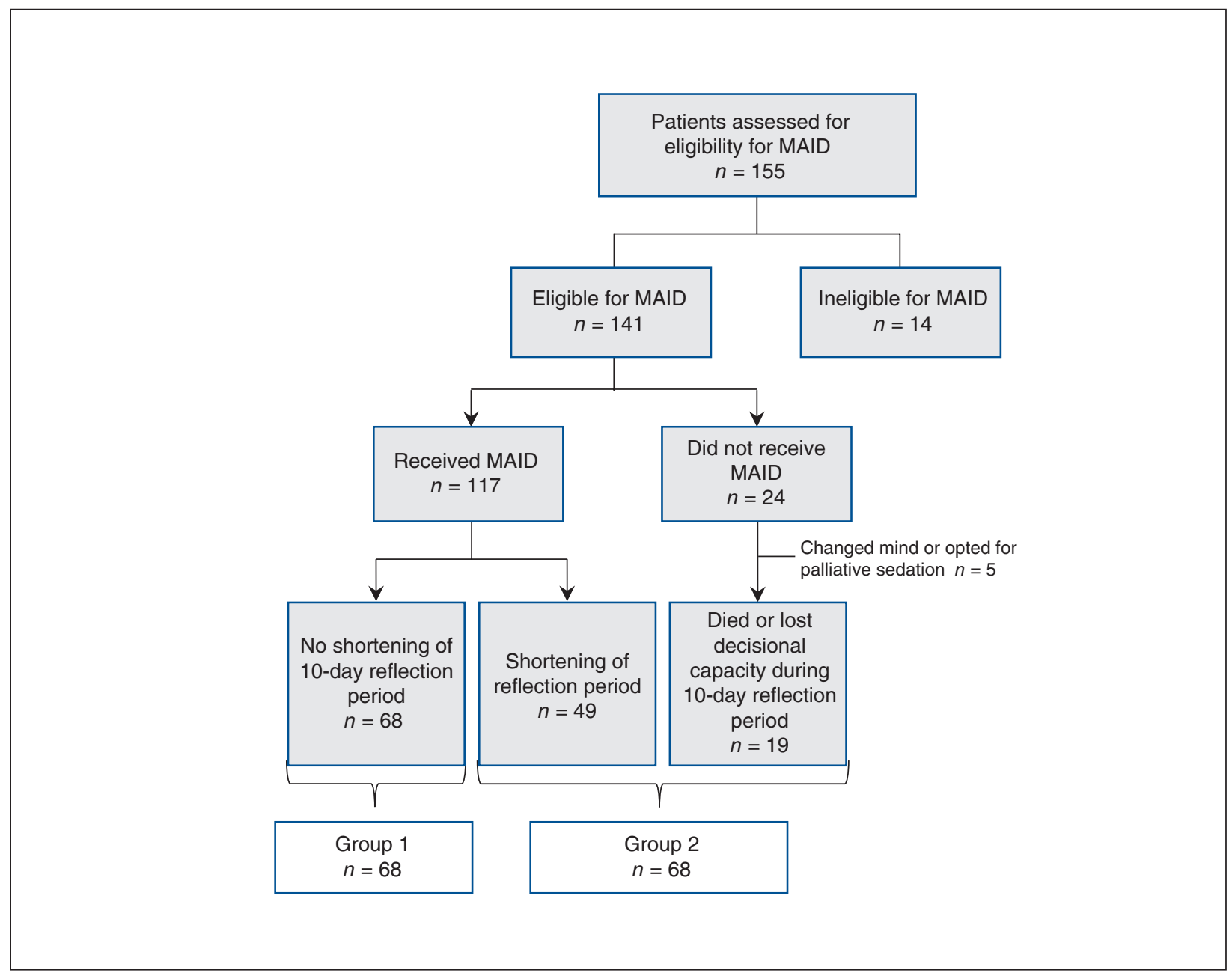

Figure 1: Outcome of all referrals for assessment of eligibility for medical assistance in dying over study period.

comparison group for those who completed the reflection period. The clinical parameters above could then be compared with the goal of identifying any that might assist clinicians in identifying higher-risk patients.

We calculated sensitivity, specificity, positive predictive value and negative predictive value for each PPS value.

We generated a receiver operating characteristic curve to further examine the relation between PPS and risk of loss of capacity.

\section{Ethics approval}

This study was approved by the Research Ethics Board at Sunnybrook Health Sciences Centre.

\section{Results}

Between June 17, 2016, and Apr. 30, 2019, the hospital's MAiD service received requests for assessments from a total of 155 patients. Of these, 141 patients were deemed eligible, with 117 ultimately receiving the procedure. Of those eligible for MAiD, 19 patients died or lost capacity to provide consent during the 10-day reflection period, and 49 patients had the reflection period shortened because of a risk of imminent loss of capacity (Figure 1). Demographic characteristics are presented in Table 1.

Age, sex and diagnosis were not significantly different between the 2 groups of interest. However, logistic regression analysis showed that a 10-point decrease in PPS score was associated with a statistically significant increase in the odds of shortening of the reflection period, loss of capacity or death within the reflection period (odds ratio 4.63, 95\% confidence interval 2.87 to 8.23 ). The location where the written request was made was significant on bivariable analysis but not multivariable analysis, where PPS was the predominant variable of significance (Table 2).

The distribution of PPS scores between the groups is shown in Figure 2, with a skewed distribution toward lower PPS values in group 2 relative to group 1.

The receiver operating characteristic curve had a high area under the curve (0.87). The point closest to 1,1 (maximizing both sensitivity and specificity) was PPS less than or equal to $30 \%$; however, a cut-off of PPS less than or equal to $40 \%$ remains proximal to 1,1 while improving sensitivity and negative predictive value (Table 3, Figure 3). 


\begin{tabular}{|c|c|c|c|}
\hline \multirow[b]{2}{*}{ Variable } & \multicolumn{3}{|c|}{ Group; no. (\%) of patients* } \\
\hline & $\begin{array}{l}\text { Group 1: Received MAiD } \\
\text { with 10-day reflection period }\end{array}$ & $\begin{array}{l}\text { Group 2a: Received MAiD } \\
\text { with shortened } \\
\text { reflection period }\end{array}$ & $\begin{array}{l}\text { Group 2b: Lost capacity } \\
\text { or died during } \\
\text { reflection period }\end{array}$ \\
\hline Total number & 68 & 49 & 19 \\
\hline Age, yr, median (range) & 73 (28 to 95$)$ & 77 (51 to 102$)$ & 79 (37 to 95$)$ \\
\hline \multicolumn{4}{|l|}{ Sex } \\
\hline Male & $35(51)$ & 19 (39) & $11(58)$ \\
\hline Female & $33(49)$ & $30(61)$ & $8(42)$ \\
\hline $\begin{array}{l}\text { PPS score at time of written MAiD request, } \\
\% \text {, median (range) }\end{array}$ & 50 (30 to 70$)$ & $30(10$ to 50$)$ & 30 (20 to 50$)$ \\
\hline \multicolumn{4}{|l|}{ Location of request } \\
\hline Inpatient & $38(56)$ & $44(90)$ & 17 (89) \\
\hline Outpatient & $30(44)$ & $5(10)$ & $2(11)$ \\
\hline \multicolumn{4}{|l|}{ Diagnosis } \\
\hline Cancer & $52(76)$ & $34(69)$ & $15(79)$ \\
\hline Noncancer & $16(24)$ & $15(31)$ & $4(21)$ \\
\hline $\begin{array}{l}\text { Time between written MAiD request and } \\
\text { first assessment, } d \text {, median (range) } \dagger\end{array}$ & $3.5(-5$ to 277$)$ & $0(-3$ to 7$)$ & $1(-1$ to 82$)$ \\
\hline
\end{tabular}

Table 2: Bivariable and multivariable logistic regression analysis of potential risk factors for loss of decisional capacity, death or shortening of 10-day reflection period for medical assistance in dying

Type of analysis; OR $(95 \% \mathrm{Cl})$

\begin{tabular}{|c|c|c|}
\hline Variable & Bivariable logistic regression & Multivariable logistic regression \\
\hline PPS score, per 10-unit decrease & $4.49(2.91-7.55)$ & $4.63(2.87-8.23)$ \\
\hline Age, continuous & $1.02(0.997-1.05)$ & $0.99(0.96-1.03)$ \\
\hline Sex, female & $1.34(0.69-2.65)$ & $1.70(0.66-4.52)$ \\
\hline Location of signing written request, inpatient & $6.88(2.89-18.46)$ & $1.99(0.65-6.41)$ \\
\hline Diagnosis, cancer & $0.79(0.36-1.71)$ & $2.19(0.71-7.14)$ \\
\hline
\end{tabular}

\section{Interpretation}

Our study identified PPS as the strongest predictor of loss of capacity or shortening of the reflection period among patients eligible for MAiD at our hospital, relative to age, sex, location (inpatient v. outpatient) and diagnosis (cancer v. noncancer). Given the value placed on MAiD by many patients, we believe that a PPS of less than or equal to $40 \%$ optimally balances both sensitivity and specificity in this population. Given that many jurisdictions internationally have mandated reflection periods, these findings may provide guidance to practitioners in assessing and following those patients who request assistance in dying.

Other jurisdictions have taken various approaches to their mandated reflection periods. In Victoria, Australia, the Voluntary Assisted Dying Act indicates that the shortest time from first physician assessment to administration of medication is 10 days, ${ }^{17}$ although this period can be shortened if both assessors agree that the patient is at risk of imminent death. Similarly, in several US states where aid in dying is legal, there is a 15-day wait period between the first and second oral requests, and a further 48-hour wait period 


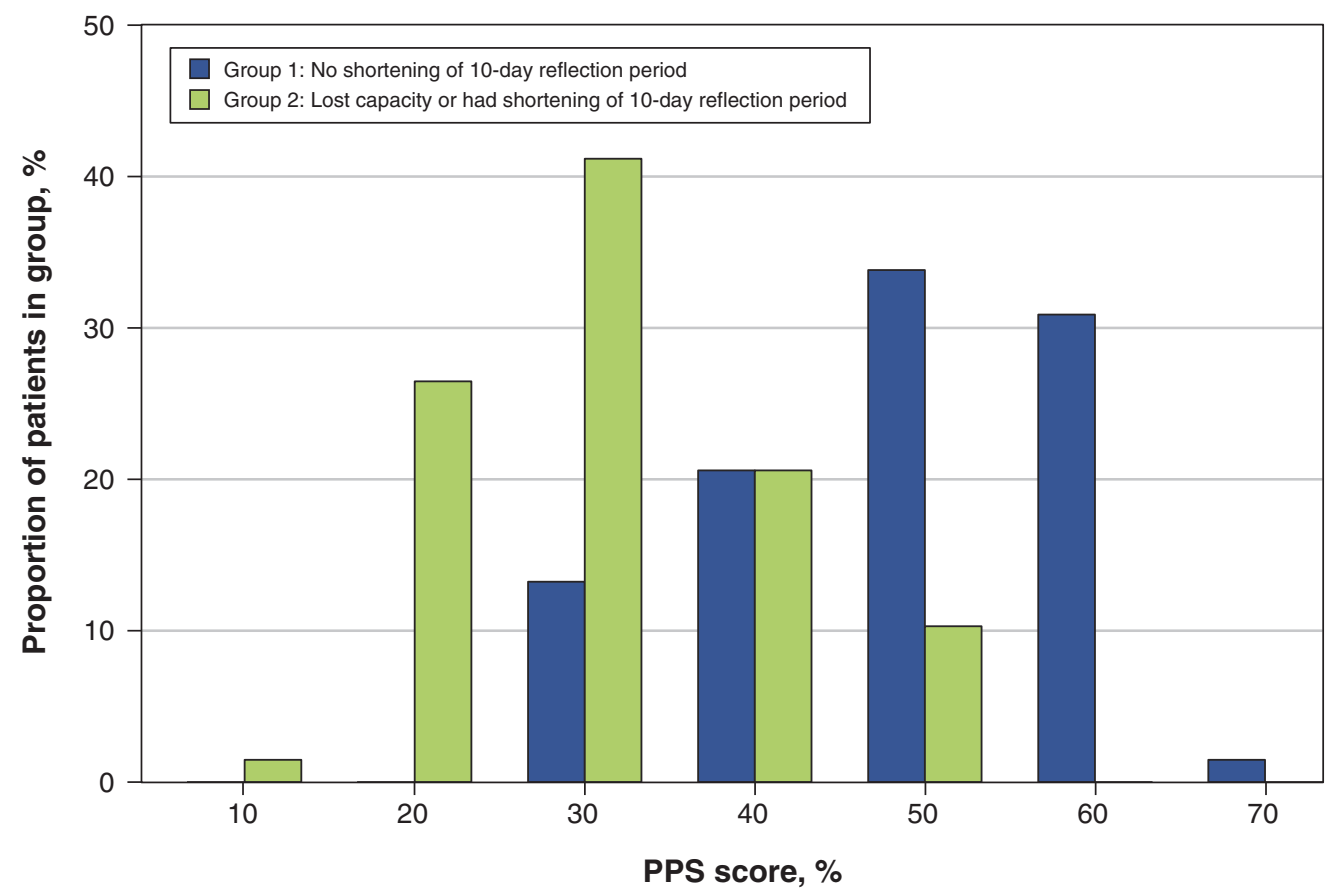

Figure 2: Distribution of palliative performance scale (PPS) scores for patients who lost decisional capacity or whose reflection period was shortened, relative to those without shortening of the reflection period.

between receipt of a written request and authorization of the prescription by a physician. ${ }^{18}$ Belgian euthanasia law requires a wait period of "at least one month between the written request and the performance of euthanasia in situations where death is not imminent." 19 However, advance directives in Belgium are also permitted; such a directive would be acted upon only in the instance of a patient who has become unconscious at the time of euthanasia. Although the Canadian law itself is now under review, and the mandatory reflection period may no longer be required in the updated legislation, identifying patients who are at high risk of a rapid change in condition remains clinically relevant.

Previously published findings from both our group and others ${ }^{12,20,21}$ have suggested that a request for MAiD is a reflection of the individual's long-standing personality characteristics, world view and lifelong values. Individuals accessing MAiD typically strongly desire control over the conditions of their end-oflife care and place importance on autonomy, independence and a sense of self. ${ }^{14,21}$ For these patients, the possibility of being denied access to MAID because of a loss of capacity is tremendously distressing, to the point that some will forgo adequate symptom management to ensure that medications do not interfere with their capacity. ${ }^{22}$ The challenge lies in identifying which patients appropriately merit exclusion from the mandated wait period while still respecting the legislative safeguards.
Three-quarters of our cohort had a primary cancer diagnosis. We anticipated that malignancy would be associated with greater risk of early loss of capacity, given the risk of precipitous decline from intercurrent complications in these patients. In comparison, patients with nonmalignant disease often live for longer periods despite substantial disability. However, we did not find that diagnosis (in terms of the binary division of cancer v. noncancer) was a significant predictor. This may be explained in part by recognizing that for both groups, decision-making about the end of life may have been left to very late stages of illness, specifically at the time of an acute decline, as opposed to being addressed earlier in the trajectory of disease.

Location of the request was significant on bivariable analysis, with admitted patients having a greater risk of shortening of the reflection period, death or loss of capacity relative to those who made their request as outpatients. However, the magnitude of this effect was attenuated on multivariable analysis, with PPS accounting for the differences seen.

PPS has previously been shown to be an independent predictor of death in various health care settings, with lower PPS scores indicating poorer function and shorter overall survival times. ${ }^{23-27}$ We observed that the distribution of PPS values differed between the 2 groups, with skewing toward lower PPS values in group 2 relative to group 1 (median 


\begin{tabular}{|c|c|c|c|c|c|}
\hline PPS, \%* & Sensitivity & Specificity & $\begin{array}{c}\text { Positive } \\
\text { predictive value }\end{array}$ & $\begin{array}{c}\text { Negative } \\
\text { predictive value }\end{array}$ & Distance from 1,1 \\
\hline$\leq 10$ & 0.015 & 1.000 & 1.000 & 0.504 & 0.985 \\
\hline$\leq 20$ & 0.279 & 1.000 & 1.000 & 0.581 & 0.721 \\
\hline$\leq 30$ & 0.691 & 0.868 & 0.839 & 0.738 & 0.336 \\
\hline$\leq 40$ & 0.897 & 0.662 & 0.726 & 0.865 & 0.354 \\
\hline$\leq 50$ & 1.000 & 0.324 & 0.596 & 1.000 & 0.676 \\
\hline$\leq 60$ & 1.000 & 0.015 & 0.504 & 1.000 & 0.985 \\
\hline$\leq 70$ & 1.000 & 0.000 & 0.500 & $-\dagger$ & 1.000 \\
\hline
\end{tabular}

PPS $30 \%$ v. 50\%; Figure 2). Although it is sometimes possible to predict a patient's imminent loss of capacity and the need for urgent provision of MAiD at the time of first assessment, our cohort included many patients for whom we did not anticipate this need initially. Further analysis of the 19 patients who lost capacity or died before provision of MAiD (group 2b) again showed a median PPS of $30 \%$ (range $20 \%-50 \%$ ), as compared with the higher median

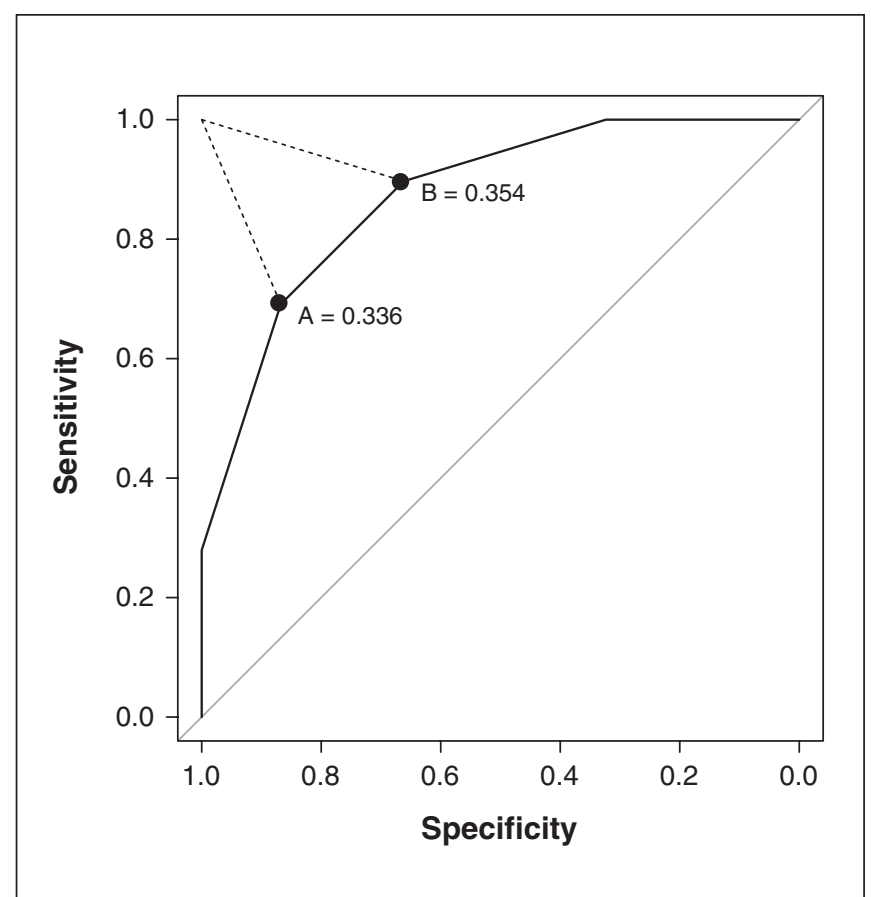

Figure 3: Receiver operating characteristic curve for palliative performance scale (PPS). Area under the curve $=0.87$. Point $A=$ cut point for PPS $\leq 30$ (where distance from optimal sensitivity/specificity is 0.336 ), with sensitivity 0.691 , specificity 0.868 , positive predictive value (PPV) 0.839 and negative predictive value (NPV) 0.738. Point $B=$ cut point for PPS $\leq 40$ (where distance from optimal sensitivity/ specificity is 0.354 ), with sensitivity 0.897 , specificity 0.662 , PPV 0.726 and NPV 0.865 .
PPS in group 1. To ensure that delayed assessment was not a factor in group $2 \mathrm{~b}$, we noted the time from completion of the formal written request to the time of first assessment. As shown in Table 1 , the median time between request and assessment was similar between groups $2 \mathrm{a}$ and $2 \mathrm{~b}$. Attention to PPS at the time of the initial request may highlight patients more vulnerable to rapid decline and in need of regular surveillance, to reduce the chances of subsequent loss of capacity and therefore loss of the opportunity to receive MAiD.

The receiver operating characteristic curve for PPS indicates that if sensitivity and specificity are considered to be equally important, a PPS of $30 \%$ or less would be the appropriate cut-off (distance to $1,1=0.336$ ). However, given the crucial importance of capturing the window for access to MAiD in these patients, we propose that the sensitivity of the test criteria carries greater importance than specificity. A PPS of $40 \%$ or less offers greater sensitivity (0.897; Table 3), resulting in a lower proportion of false-negative errors, while still remaining proximal to 1,1 (distance $=0.354)$. As such, we propose using a PPS value of less than or equal to $40 \%$ at the time of first assessment as a potential marker for patients at high risk of early loss of capacity or death.

With this knowledge in mind, we have adapted our institutional approach to mitigate the risk of patients losing capacity before provision of MAiD, specifically when they have identified MAiD as being of high importance. For any patient with a PPS score less than or equal to $40 \%$ at the time of first assessment, the primary MAiD assessor follows the patient closely, usually with daily assessments, with a view to identifying any clinical deterioration. The patient and family are alerted at the outset to the strong possibility that MAiD may need to proceed on an urgent basis. The vascular access team assesses the patient promptly to ensure adequate venous access, and the pharmacy is notified. The unit's patient care manager and interdisciplinary staff are notified of the possibility of urgent provision of MAiD, so that appropriate staffing can be allocated for the procedure and bereavement support. 


\section{Limitations}

The main limitation of this study was the use of a predominantly inpatient population in a single clinical setting; as such, the results may not be generalizable to other patient populations or other settings. In particular, predictive values may be higher in our sample than in a sample from a largely outpatient setting. Furthermore, the number of assessors at our institution was limited, which may have added individual bias to clinical decisions that would differ from those of other assessors. Similar analyses at other sites would be warranted to confirm or refute these findings.

\section{Conclusion}

Unlike age, sex, location of request and diagnosis, our study showed that the PPS score was associated with loss of capacity or shortening of the reflection period, with lower scores incrementally increasing the risk of these outcomes. These findings suggest that when patients with PPS less than or equal to $40 \%$ have made a formal request for MAiD, they should be followed carefully, potentially with steps taken to allow for rapid provision of MAiD and with recognition of the potential need to shorten the reflection period. Further research should seek to validate the utility of the proposed PPS cut-off value externally, particularly in different patient care settings, as well as different institutions. In addition, exploring the impact of a changing PPS may further help in identifying those at risk of losing decisional capacity.

\section{References}

1. Carter v. Canada (Attorney General) 2015 SCC 5. 1 SCR 331 [Carter]

2. Criminal Code, R.S.C., 1985 , c. C-46.

3. First annual report on medical assistance in dying in Canada 2019. Ottawa: Government of Canada; modified 2020 July 24. Available: https://www.canada.ca/ en/health-canada/services/medical-assistance-dying-annual-report-2019.html (accessed 2020 Aug. 25).

4. Chin AE, Hedberg K, Higginson GK, et al. Legalized physician-assisted suicide in Oregon - the first years' experience. N Engl 7 Med 1999;340:577-83.

5. The Washington Death with Dignity Act. Chapter 70.245 RCW. Olympia (WA): Washington State Legislature. Available: https://app.leg.wa.gov/rcw/default. aspx? cite $=70.245$ (accessed 2019 Oct. 12).

6. Watson R. Luxembourg is to allow euthanasia from 1 April. BM7 2009;338: b1248.

7. Republic of Colombia Constitutional Court, Sentence \# c-239/97. Ref. Expedient \# D-1490, May 20, 1997.

8. Deliens L, van der Wal G. The euthanasia law in Belgium and the Netherlands. Lancet 2003;362:1239-40.

9. Buiting H, van Delden J, Onwuteaka-Philpsen B, et al. Reporting of euthanasia and physician-assisted suicide in the Netherlands: descriptive study. $B M C$ Med Ethics 2009;10:18.

10. Regulations for the monitoring of medical assistance in dying: SOR/2018-166. Canada Gazette Part II 2018;152:16. Available: www.gazette.gc.ca/rp-pr/ p2/2018/2018-08-08/html/sor-dors166-eng.html (accessed 2019 Oct. 13).

11. Provincial and territorial contact information for end of life care services. Ottawa: Government of Canada; 2018. Available: www.canada.ca/en/health-canada/ services/provincial-territorial-contact-information-links-end-life-care.html (accessed 2019 Oct. 18).
12. Selby D, Bean S, Isenberg-Grzeda E, et al. Medical assistance in dying (MAiD): a descriptive study from a Canadian tertiary care hospital. Am 7 Hosp Palliat Care 2020;37:58-64.

13. Hales B, Bean S, Isenberg-Grzeda $\mathrm{E}$, et al. Improving the medical assistance in dying (MAID) process: a qualitative study of family caregiver perspectives. Palliat Support Care 2019;17:590-5.

14. Nuhn A, Holmes S, Kelly M, et al. Experiences and perspectives of people who pursued medical assistance in dying. Can Fam Physician 2018;64: e380-6.

15. Anderson F, Downing GM, Hill J, et al. Palliative performance scale (PPS): a new tool. 7 Palliat Care 1996;12:5-11.

16. Olajide O, Hanson L, Usher B, et al. Validation of the palliative performance scale in the acute tertiary care hospital setting. 7 Palliat Med 2007; 10:111-7.

17. Voluntary assisted dying - information for people considering voluntary assisted dying. Melbourne (AU): Department of Health \& Human Services; 2019. Available: https://www2.health.vic.gov.au/about/publications/policiesandguidelines/ information-for-people-considering-voluntary-assisted-dying (accessed 2020 Nov. 26).

18. Frequently asked questions. Portland (OR): Dying with Dignity. Available: www.deathwithdignity.org/faqs/ (accessed 2019 Oct. 13).

19. Nicol J, Tiedemann M. Euthanasia and assisted suicide: the law in selected countries [background paper]. Ottawa: Library of Parliament; 2015. Publication no. 2015-116-E.

20. Smith KA, Harvath T, Goy E, et al. Predictors of pursuit of physicianassisted death. 7 Pain Symptom Manage 2015;49:555-61.

21. Oldham RL, Dobscha SK, Goy ER, et al. Attachment styles of Oregonians who request physician-assisted death. Palliat Support Care 2011;9:123-8.

22. Downie J, Chandler JA. Interpreting Canada's medical assistance in dying legislation. Montréal: Institute for Research on Public Policy; 2018. Available: https://irpp.org/research-studies/interpreting-canadas-medical-assistance-in -dying-maid-legislation/ (accessed 2019 Oct. 13).

23. Selby D, Chakraborty A, Lilien T, et al. Clinician accuracy when estimating survival duration: the role of the patient's performance status and time-based prognostic categories. 7 Pain Symptom Manage 2011;42:578-88.

24. Myers J, Kim A, Flanagan J, et al. Palliative performance scale and survival among outpatients with advanced cancer. Support Care Cancer 2015;23:913-8.

25. Lau F, Downing $M$, Lesperance $M$, et al. Use of palliative performance scale in end-of-life prognostication. 7 Palliat Med 2006;9:1066-75.

26. Downing M, Lau F, Lesperance $M$, et al. Meta-analysis of survival prediction with palliative performance score. 7 Palliat Care 2007;23:245-52.

27. Olajide $\mathrm{O}$, Hanson L, Usher B, et al. Validation of the palliative performance scale in the acute tertiary care hospital setting. 7 Palliat Med 2007;10:111-7.

Affiliations: Sunnybrook Health Sciences Centre (Selby, Bean, IsenbergGrzeda, Nolen); Department of Family and Community Medicine (Selby, Meaney, Nolen), Dalla Lana School of Public Health (Bean) and Department of Psychiatry (Isenberg-Grzeda), University of Toronto, Toronto, Ont.

Contributors: Debbie Selby, Sally Bean, Amy Nolen and Elie IsenbergGrzeda contributed to study concept, designed the study and acquired the data. Christopher Meaney performed the statistical analysis and data interpretation. All of the authors contributed to drafting the manuscript, revised the manuscript for important intellectual content, approved the final version for publication and agreed to be accountable for the work.

Funding: Funding to cover the costs of statistical analysis and publication were provided by the research fund of the Division of Long Term Care at Sunnybrook Health Sciences Centre.

Data sharing: The data used in this study are not available to others at present.

Supplemental information: For reviewer comments and the original submission of this manuscript, please see www.cmajopen.ca/content/8/4/ E825/suppl/DC1. 